\title{
Classifying Stem Cell Differentiation Images by Information Distance
}

\author{
Xianglilan Zhang^, Hongnan Wang, Tony J. Collins, \\ Zhigang Luo, and Ming $\mathrm{Li}^{\star \star}$ \\ School of Computer, National University of Defense Technology, \\ Changsha, Hunan 410072, China \\ David R. Cheriton School of Computer Science, University of Waterloo, \\ Waterloo, Ontario N2L 3G1, Canada \\ Stem Cell and Cancer Research Institute, Mcmaster University, \\ Hamilton, Ontario L8S 4K1, Canada \\ \{x322zhang,mli\}@uwaterloo.ca, \\ WangHongnan1979@yahoo.com, \\ tjc@mcmaster.ca, \\ zgluo@nudt.edu.cn
}

\begin{abstract}
The ability of stem cells holds great potential for drug discovery and cell replacement therapy. To realize this potential, effective high content screening for drug candidates is required. Analysis of images from high content screening typically requires DNA staining to identify cell nuclei to do cell segmentation before feature extraction and classification. However, DNA staining has negative effects on cell growth, and segmentation algorithms err when compound treatments cause nuclear or cell swelling/shrinkage. In this paper, we introduced a novel Information Distance Classification (IDC) method, requiring no segmentation or feature extraction; hence no DNA staining is needed. In classifying 480 candidate compounds that may be used to stimulate stem cell differentiation, the proposed IDC method was demonstrated to achieve a $3 \%$ higher $F_{1}$ score than conventional analysis. As far as we know, this is the first work to apply information distance in high content screening.
\end{abstract}

Keywords: information distance, stem cell differentiation image classification, compound classification.

\section{Introduction}

Stem cells are characterized by their ability to transform, by a process referred to as differentiation, from a primitive pluripotent state into diverse specialized mature cell types, and to self-renew to produce more pluripotent stem cells [1]. Various tissues, such as muscles and nerves, are grown and transformed from stem cells. In current stem cell research, compounds that will induce this differentiation are being sought

\footnotetext{
* Xianglilan Zhang is currently a visiting Ph.D. student at David R. Cheriton School of Computer Science of University of Waterloo (from October 2010 to October 2012).

${ }^{\star \star} \mathrm{Ming} \mathrm{Li}$ is the corresponding author.
}

P. Flach et al. (Eds.): ECML PKDD 2012, Part I, LNCS 7523, pp. 269-282, 2012.

(C) Springer-Verlag Berlin Heidelberg 2012 
often through high content screening. High content screening, whereby thousands of compounds are tested for the effect on cells, is a key approach to the identification and development of chemical-based therapeutics that serve as tools for replacement therapy and as novel drugs for the treatment of degenerative diseases.

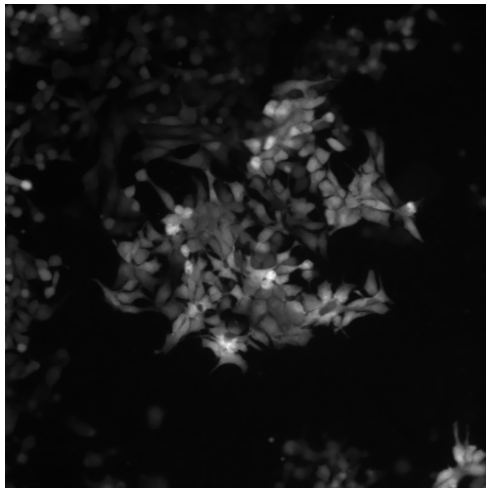

(a) High Control Compound

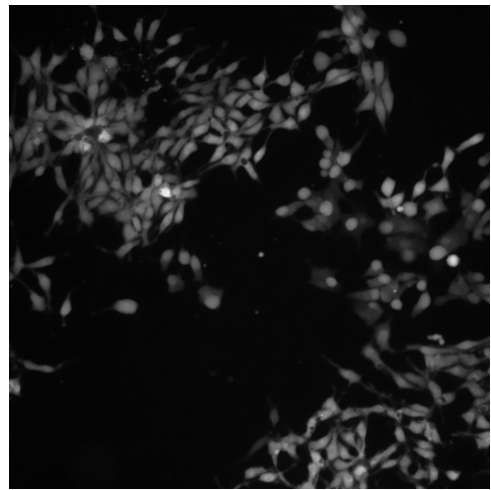

(b) Low Control Compound

Fig. 1. Cluster illustration. Each image represents a different type of cluster. Fig. 11(a) shows the cell situation after a high control compound is added; Fig. 1)(b) shows the cell situation after a low control compound is added. Theoretically, the cells treated by a high control compound should become larger in size and decrease more in number than cells treated by a low control compound.

Primary high content screening is time-consuming and expensive, and secondary assays to validate the primary screening are also generally complex. This is especially true for stem cell biology where expansion of undifferentiated cells is technically more challenging. Thus, efficient and accurate computer-assisted compound classification is critical for efficient secondary biological compound identification. Our goal is to analyze images of human embryonic stem cells expressing a green fluorescent protein (GFP)-based pluripotency reporter and thus to identify those compounds that induce the cells to differentiate. Upon differentiation with a known differentiation treatment (BMP4; high control), the cells show reduced GFP fluorescence intensity, are reduced in numbers, and appear larger. As shown in Fig. 1, a high control compound means a known active compound that can cause stem cell differentiation; a low control compound is a known inactive compound that does not induce stem cell differentiation. If a compound tested is classified with the high control cluster, it can be treated as an active compound; if it is classified with the low control cluster, it can be treated as an inactive compound. Through stem cell differentiation image classification, we can label the presumed active compounds, thus decreasing the number of active compound candidates. Activity can be confirmed with a dose-effect experiment. The more accurately and 
efficiently active compounds are labeled, the cheaper and faster the biological verification step will be.

In the above assay, compounds are typically classified based on the GFP level in cells. In the classical approaches [3] (i.e., conventional univariate analysis and multivariate analysis), a DNA stain is added to label cell nuclei. Nuclear segmentation algorithms are then fine-tuned to identify individual nuclei, which are presumed to represent individual cells. These nuclei are then used as foci to identify and segment the cytoplasmic area. Most of the DNA stains are cytotoxic or at least cytostatic, and have a negative impact on cell growth. Moreover, the need to set constraints on the segmentation algorithms, such as maximum/minimum size, means segmentation errors when compound treatments cause nuclear or cell swelling/shrinkage.

Therefore, we introduce a novel method, the Information Distance Classification (IDC) method, to classify compounds, whereby we analyze the similarity of stem cell differentiation images from each well containing one unique compound. Unlike traditional methods, ours does not involve segmentation, and thus does not require DNA staining. The method depends on information distance, based on Kolmogorov complexity [4], first introduced by [5], and has been applied to many different areas, from image processing to weather broadcasting, to software engineering, and to bioinformatics [6-27]. This method skips the feature-extraction step in practice. Therefore, it does not need manual intervention, as required in conventional analysis, to define the cell count and brightness for compound classification. Using our IDC method, we achieve results comparable to those of conventional analysis. To the best of our knowledge, our paper is the first to utilize information distance for a novel application in high content screening.

\section{Information Distance}

The classification of stem cell differentiation images essentially depends on determining whether the cells in an image are "similar" to those acquired from high or low control compounds. Conventional methods involving segmentation to acquire cell information for extracting features is just one way to measure image similarity. We want to find a general and optimal approach for measuring the similarity between two cell images.

In the early 1990s, in [5], the authors studied the energy cost of conversion between two strings, $x$ and $y$. John von Neumann hypothesized that performing 1 bit of information processing costs $1 K_{B} T$ of energy, where $K_{B}$ is the Boltzmann's constant and $T$ is the room temperature. In the 1960 s, observing that reversible computations can be done for free, Rolf Landauer revised von Neumann's proposal to hold only for irreversible computations. Starting from this von Neuman-Landauer principle, [5] proposed using the minimum number of bits needed to convert between $x$ and $y$ to define their distance. Formally, with respect to a universal Turing machine $U$, the cost of conversion between $x$ and $y$ is defined as:

$$
E(x, y)=\min \{|p|: U(x, p)=y, U(y, p)=x\}
$$

It is clear that $E(x, y) \leq K(x \mid y)+K(y \mid x)$. In [5] the following optimal result was obtained, up to an additive factor of $O(\log (|x|+|y|))$ : 
Theorem 1. $E(x, y)=\max \{K(x \mid y), K(y \mid x)\}$.

Here $K(x \mid y)$ is the Kolmogorov complexity [4] of a binary string $x$ condition to another binary string $y, K(x \mid y)$, which is informally defined to be the length of the shortest program that outputs $x$ with input $y$. We refer the readers to [4] for further details of Kolmogorov complexity and its rich applications in computer science and many other disciplines.

This complexity has enabled us to define the information distance between two sequences $x$ and $y$ as:

$$
D_{\max }(x, y)=\max \{K(x \mid y), K(y \mid x)\} .
$$

This distance is shown to satisfy the basic distance requirements such as positivity, symmetricity, and triangle inequality. Furthermore, $D_{\max }$ is "universal" in the following sense. A distance $D$ is admissible if

$$
\sum_{y} 2^{-D(x, y)} \leq 1
$$

$D_{\max }(x, y)$ satisfies the above requirement because of Kraft's Inequality (with the prefix-free version of Kolmogorov complexity). It was proved in [5] that for any admissible computable distance $D$, there is a constant $c$, for all $x, y$ :

$$
D_{\max }(x, y) \leq D(x, y)+c .
$$

Thus, if any such distance $D$ discovers some similarity between $x$ and $y$, so will $D_{\max }$. Therefore, $D_{\max }$ is universal.

According to this theory, given two images $x$ and $y$, the ultimate distance between them is $D_{\max }(x, y)$. That is, if there is another way of finding whether $x$ and $y$ are similar, then $D_{\max }$ finds it too. The only problem is that $K(x \mid y)$ is known to be uncomputable. Nevertheless, for practical applications, universal compression algorithms can be used to approximate $K(x \mid y)$ :

$$
D_{\max }(x, y)=\max \{C(x \mid y), C(y \mid x)\} .
$$

where $\mathrm{C}$ is a compression algorithm.

This approach was first introduced by [20] to measure the information distance between two genomes. Many other applications in various different fields have been found. Recently, it was used in [8] and [21] to measure the similarity of images, and both studies obtained promising results.

\section{Materials}

In this section, we describe the image data acquisition process. Images from the OCRiT v1O4 primary screening of the NIH clinical compound collection were used to develop the stem cell differentiation analysis. As shown in Fig. 2, this collection equated to six library plates run in triplicate to become 18 assay plates. Each plate had 96 wells in 
chemical collection, a.k.a. 'library'

e.g.NGPOO3

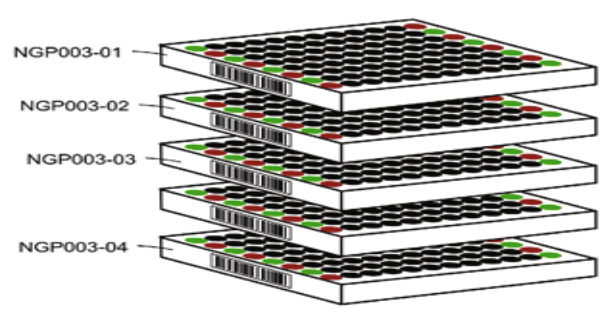

Images acquired of each plate and saved to a single folder with unique identifier ID\#

e.g. NGP003-01-date-replicate\#[ID\#]

e.g. NGP003-01-110117-[1210] single library plate of compounds plus control treatments in end columns

a.k.a 'master plate'

e.g.NGP003-01

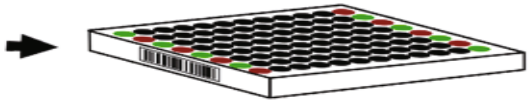

Each well of master plate added to three plates with cells in for quantification.

a.k.a 'assay plate'

e.g. NGP003-01-date-replicate\#

e.g. NGP003-01-110117-1

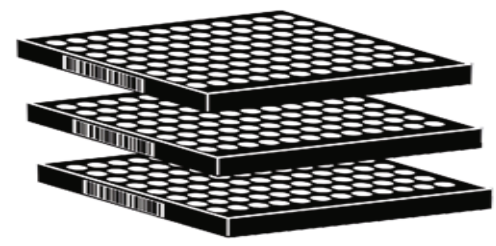

Fig. 2. Assay workflow. Single library plate contained three assay plates. Each plate had control treatments in its end columns. The images of each plate were captured and then stored in a single folder with a unique ID number.

total, and each well contained a different compound at $10 \mu \mathrm{M}$ concentration. Eight high and eight low control compounds were alternated in the columns at either end of the plates. Here, high control means known active, and low control means known inactive. Thus, each plate had 80 compounds tested.

Human stem cells expressing GFP pluripotency reporters were added to each well to show the cytoplasm of cells. At the end of the experiment, the DNA stain, Hoechst, was added to visualize the cell nuclei. Two fluorescent images (GFP and Hoechst) were acquired for nine fields per well. Since each compound was sampled three times, this totaled 27 images per compound.

It should be noted that, because IDC analysis does not require segmentation, only the GFP images were analyzed. Using IDC would have removed the need for any preacquisition sample processing apart from a media wash to remove the autofluorescent growth medium. 


\section{Information Distance Classification Method}

Here, we propose our IDC method to describe the process of compound classification.

It is important to note that scattering GFPs over the cell culture wells causes uneven illumination and a "speckle" effect outside of the cells. To remove these illumination inconsistencies and noises in images, we change Eq. 5 to the following:

$$
D_{\max }(x, y)=\min \{\max \{C(x \mid y), C(y \mid x)\}, \max \{C(f(x) \mid f(y)), C(f(y) \mid f(x))\}\} .
$$

where

$$
\begin{gathered}
f(I)=\frac{I-\min (I)}{\max (I)-\min (I)} \\
\min _{I}\left\{\iint_{\Omega} \sqrt{I_{x}^{2}+I_{y}^{2}} \mathrm{~d} x \mathrm{~d} y+\frac{1}{2} \iint_{\Omega}\left(I-I_{0}\right)^{2} \mathrm{~d} x \mathrm{~d} y\right\}
\end{gathered}
$$

$I$ is the denoising result of the total variation method [28]. $\Omega$ is an image domain. $I_{x}$ and $I_{y}$ are the first-order partial derivatives of image $I$ with respect to $x$ and $y$ directions. $I_{0}$ is the original image.

Generally speaking, this method contains seven steps:

1. Use function $f$ in Eq. 6 to do image preprocessing.

2. Use MPEG encoder to compress original and transformed images.

3. Use information distance to measure the distance between any pair of images.

4. Do information distance statistics on the high/low control images that represent high/low control compounds.

5. Calculate the average information distance between any image of the compound tested and the control images.

6. Classify the images.

7. Classify the compounds based on the image classification results.

Steps 2 to 7 of our method are described in greater detail in the following subsections.

\subsection{Image Compression}

MPEG is a state-of-the-art video compressor. It is appropriate for different applications because it supports spatial and temporal redundancy reduction resolution [30], is widely available, and has also highly optimized implementations [8]. According to our application, we need to find morphologically similar cells rather than identical ones. As MPEG is a lossy compressor and considering its advantages, we utilize MPEG encoder as our compressor. Using the MPEG encoder, we can set an image pixel search range according to cells' average size, which guarantees isolating a single cell from other cells in an image and acquire the cell's information of shape, size, brightness, etc. Thus, the information of two cell images is accurately compressed.

To measure the similarity of two images, the MPEG encoder creates a synthetic "video" of the two images. In this video, the first image is treated as a reference $(R)$ frame, and the second image as a predicted $(P)$ frame. Therefore, the MPEG encoder 
can provide a total combined compressed size of the pair of images. If we use two images that are the same as input, this algorithm returns the compressed size of a single image; if we use two different images as input, the MPEG encoder will give the "between frames" (two images' inter-frame compression) compressed size plus the single frame (the first image's intra-frame compression) size. Several parameters must be set on the MPEG encoder. Taking [8] as a reference, a logarithmic $P$ frame search algorithm is used for speed and consistency, the original images for intra-picture reference frames are used for the encoding step; and a bidirectional $(B)$ frame quantization factor is ignored because no bidirectional frames are used in our method. Since the image size is $512 * 512$ pixels, the integer search radius is set to 255 . Because the average cell size is about 17 pixels, the quantization scales for $R$ and $P$ frames are set to 17 to maintain image compressibility and quality.

\subsection{Image Information Distance Calculation}

According to Eq. 6, our IDC method then uses the MPEG compressed image file size to compute the image information distance. Since the MPEG encoder returns the combined compressed size of a pair of images and the first image, the total combined compressed size of the pair of images must be represented by the compressed size of the two different images minus the size of the first image.

\subsection{Control Images Statistics}

Our IDC method uses control images from the wells of control treatments, as our training set. The whole batch of images has two known clusters, one labeled high control and one low control. It is assumed that the high/low control cluster contains $n$ images indexed from 1 to $n$, and $x$ and $y$ are any two images within the same cluster. This method computes the mean value $(\mu)$ and standard deviation $(\sigma)$ of the high/low control cluster, based on the information distance between any $x$ and $y$.

\subsection{Average Image Information Distance between Any Image and Control Images}

The information distance between any image and any control image is calculated for image classification. The "any" image is indexed by $x$; $H C$ means high control images, and $L C$ means low control images. First, all the information distances between $x$ and any control image $\left(H C\left(i_{j}\right)\right.$ or $\left.L C\left(i_{j}\right),(j \in[1, n])\right)$ are calculated. To avoid the impact of extreme values, a geometric mean is chosen to represent the average value of information distances in this step. The average information distance between image $x$ and all the high control $(H C)$ images or low $\operatorname{control}(L C)$ images is represented by geomean $(I D(x, H C))$ or geomean $(I D(x, L C))$. The geomean $(I D(x, H C))$ means the geometric mean of the information distance sequence $I D(x, H C)$, and geomean $(I D(x, L C))$ means the sequence $I D(x, H C)$ where

$$
\begin{aligned}
I D(x, H C) & =I D\left(x, H C\left(i_{1}\right)\right), I D\left(x, H C\left(i_{2}\right)\right), \ldots, I D\left(x, H C\left(i_{n}\right)\right) \\
I D(x, L C) & =I D\left(x, L C\left(i_{1}\right)\right), I D\left(x, L C\left(i_{2}\right)\right), \ldots, I D\left(x, L C\left(i_{n}\right)\right)
\end{aligned}
$$




\subsection{Image Classification}

After the 4.3 Control Images Statistics step, there are two sets of mean values and standard deviations. One is $\mu_{H H}$ and $\sigma_{H H}$, which represent the mean value and standard variation of distance between any pair of high control images. The other is $\mu_{L L}$ and $\sigma_{L L}$, which show the mean value and variation of distance between any pair of low control images. Algorithm 1 classifies image $x$ to either the high control or the low control cluster, according the values of geomean $(I D(x, H C))$, geomean $(I D(x, L C))$, $\mu_{H H}, \sigma_{H H}, \mu_{L L}, \sigma_{L L}$. As shown in Fig. 3, the distribution of distances between any two high/low control images is very similar to Gaussian distribution. Therefore, we use Gaussian distribution to model the distance distribution. If the average compressed size of image $x$ and high control images is more similar to that of any two high control images, then image $x$ will be classified as high control, and vice versa.

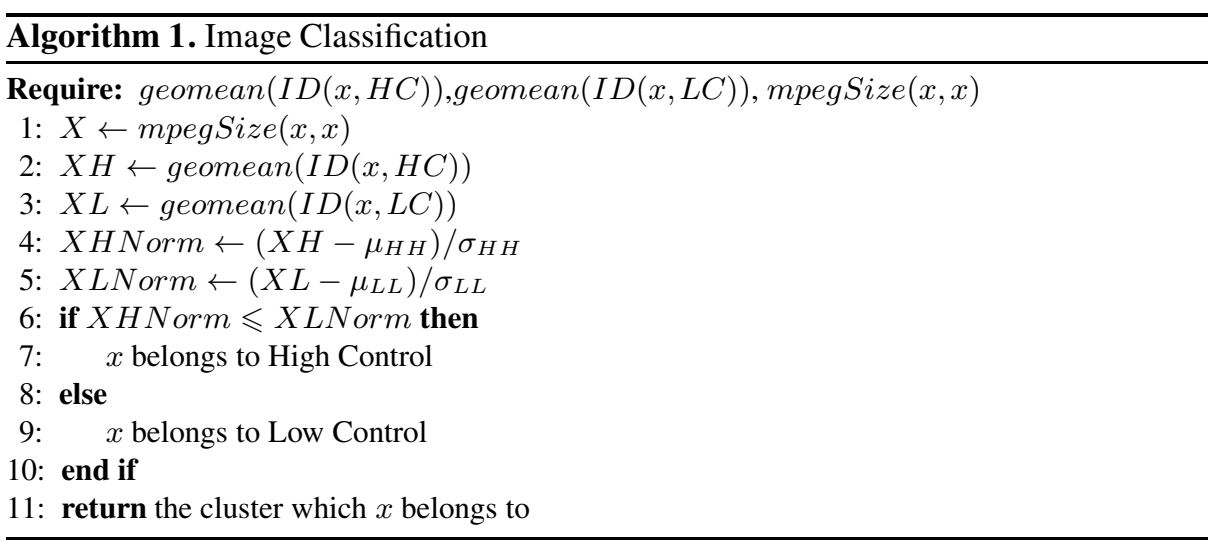

It may be asked why no between-classes measurements are computed. Since our purpose is to classify test images into either high control or low control classes, we do not consider the distance between the two. That is, there is no need to compute $\sigma_{H L}$ and $\mu_{H L}$.

\subsection{Compound Classification}

Each compound is classified based on the information distances between its images. It is assumed that there are $m$ high control compounds. $N u m_{i}$ is the number of labeled high control images contained in the $i^{\text {th }}$ high control compound. Num tested is the number of labeled high control images contained in a compound tested. Compounds tested are classified into active if the Num $_{\text {tested }}$ is larger than $\min _{i} N u m_{i}$.

All active compounds are identified after the above seven steps. Our method treats images as input, and gives the classification result directly. Based on an developed image similarity measure, our method utilizes the MPEG encoder with a fixed set of encoder-parameters to analyze GFP (cytoplasm) images and does not need Hoechst (nucleus) images. Therefore it avoids cytotoxic DNA staining, segmentation, and feature extraction steps. 


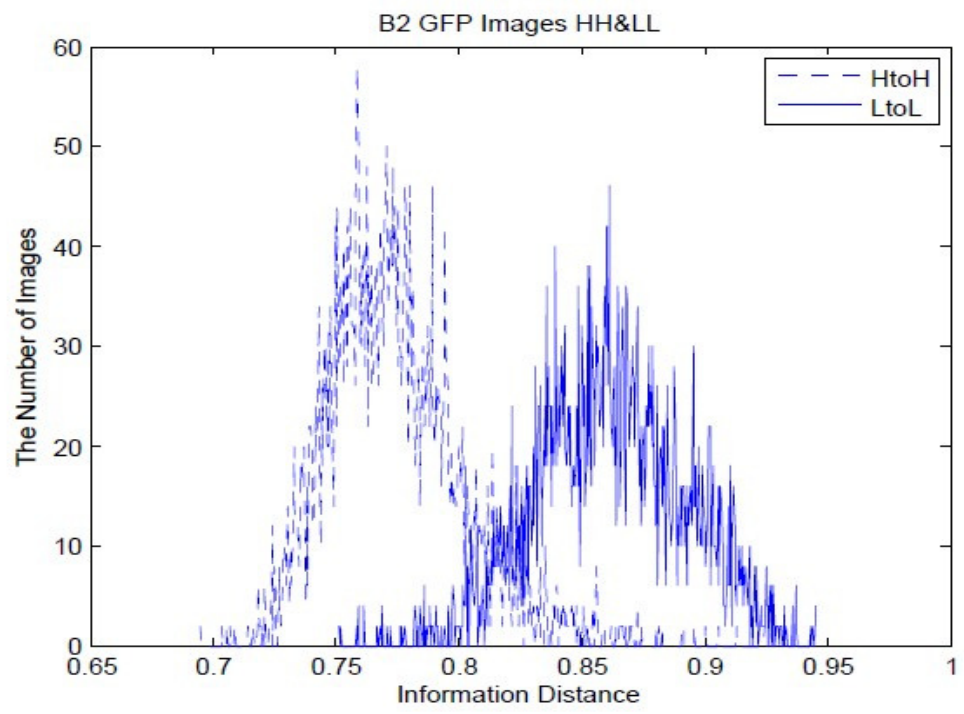

Fig. 3. Control image information distance distribution. They are the high and low control image information distance distributions of assay plate 2 of library plate 2. HtoH represents the information distance between any two high control images; and LtoL represents the information distance between any two low control images.

\section{Results}

In this section, we evaluate the effectiveness of the proposed framework for compound classification, using $\mathrm{F}_{1}$ score as our performance measure. It is defined as in Eq. 8.

$$
F_{1}=\frac{2 * T P}{2 * T P+F P+F N}
$$

where

$$
\begin{aligned}
& \text { TP : the number of true positive compounds; } \\
& F P: \text { the number of false positive compounds; } \\
& F N: \text { the number of false negative compounds. }
\end{aligned}
$$

We started by comparing the images of high and low control compounds. For short, we call these images high and low control images, respectively. Fig. 4 shows the information distance distribution of all six plates' high and low control images. The information distances between any pair of low control images were significantly larger than those between any pair of high control images.

Then we applied our IDC method to all images of the six plates' compounds. Using our method, six compounds in plate 1 , five compounds in plate 2 , six compounds in plate 3 , three compounds in plate 4 , seven compounds in plate 5 , and six compounds in plate 6 were recognized as active compounds. All of the other compounds were treated 
as inactive compounds. Fig. 5 indicates image samples taken randomly from both the active compounds (a) and inactive compounds (b). We referred to [29], and used an established commercial high content screening image analysis software - Acapella from PerkinElmer company [31] - to do the conventional analysis. Both methods in total identified 34 compounds as active; the other 446 compounds were inactive.

Those compounds flagged as active by either type of analysis were validated by further biological verification experiments. A full concentration (10 point dose) response curve for each compound was tested in 30 wells (10 concentrations in triplicate), and activity was considered "true" if the EC50 of the compound was less than $10 \mu \mathrm{M}$. For the chosen compounds tested, the biological verification experiments follow the same process as the primary screening. Since the primary screening needs one week to culture cells, the verification experiments need another week, and the same monetary cost is involved as in the primary screening to test each chosen compound.

Table 1. Biological verification results on the conventional analysis and the IDC method

(a) Conventional Analysis Statistics

\begin{tabular}{|c|l|l|}
\hline & \multicolumn{2}{|c|}{ Biological Experiment } \\
\hline Conventional & TruePositive $=29$ & FalsePositive=5 \\
\cline { 2 - 3 } Analysis & FalseNegative $=3$ & TrueNegative=9 \\
\hline
\end{tabular}

(b) IDC Statistics

\begin{tabular}{|c|c|c|}
\hline & \multicolumn{2}{|c|}{ Biological Experiment } \\
\hline Information & TruePositive $=29$ & FalsePositive= 3 \\
\cline { 2 - 3 } Distance & FalseNegative $=3$ & TrueNegative $=11$ \\
\hline
\end{tabular}

Due to the high related costs, the biological verification experiments did not test all compounds but focused mainly on those flagged as active by one or more computerassisted methods to confirm whether those identified as positives were true positives. A few other biological verification experiments were run on compounds that were flagged as negatives to confirm whether they were true negatives.

In practice, the biological verification experiment picked 46 compounds from the total 480 . These 46 compounds contain the total 34 active ones identified by the conventional analysis or the IDC method, or both. Table 2 shows the active compound identification results of the biological verification experiment, the conventional analysis and the IDC method. Table 1 shows the biological verification results for the conventional analysis and the IDC method using the 46 chosen compounds.

The $\mathrm{F}_{1}$ scores of the conventional analysis and the IDC method are 0.88 and 0.91 , respectively. Our IDC method achieves a $3 \%$ higher $F_{1}$ score than conventional analysis.

It may be perceived that the biological verification experiment may be biased, because it chose only 46 compounds that had already been shown to contain the 34 active compounds identified by union of the above two methods. However, this bias will not impact the comparison result, because it will only change those two negative values. While the absolute values of sensitivity and specificity will be changed as the total number of compounds confirmed is increased, the relative order of the sensitivity and specificity values of the two methods will not be changed. 

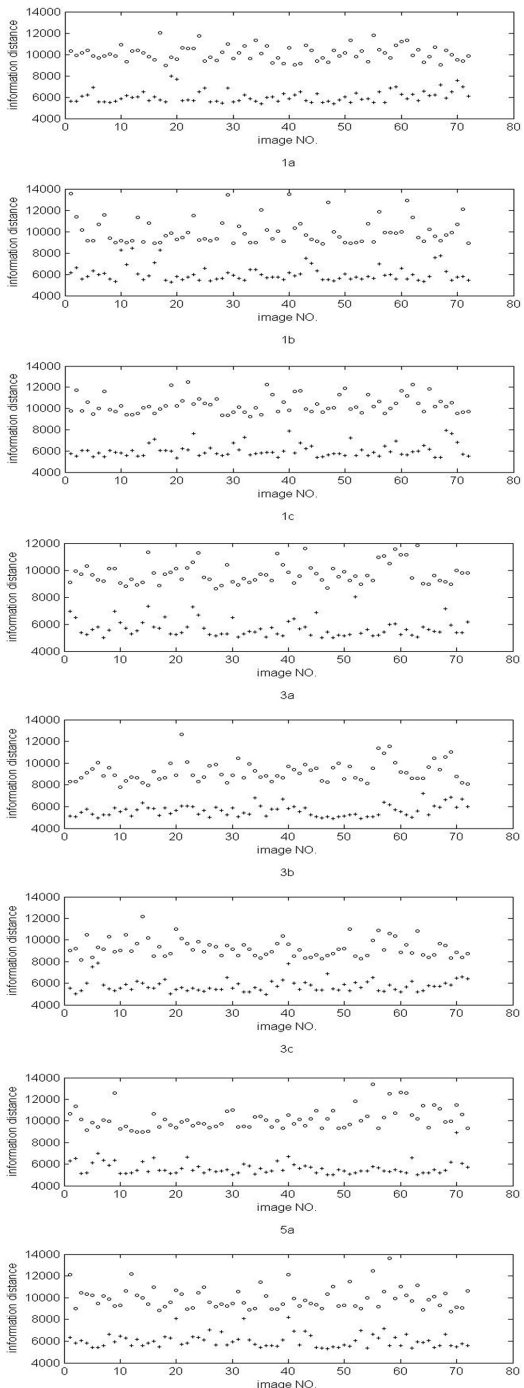

$5 \mathrm{~b}$

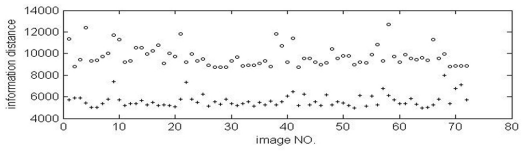

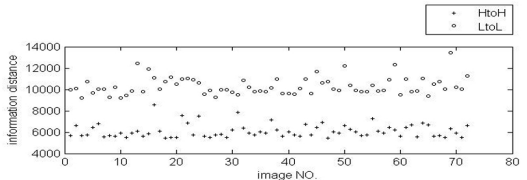

2a
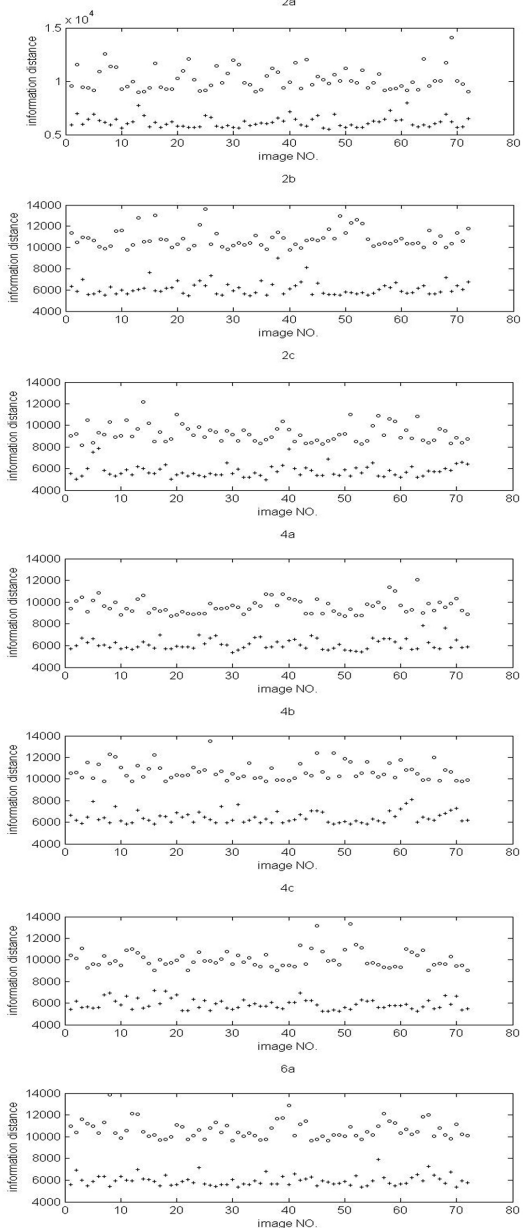

$6 \mathrm{~b}$

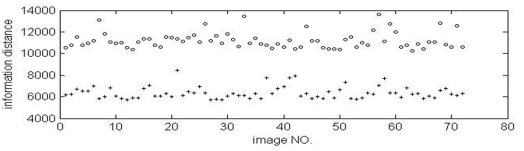

Fig. 4. The distribution of information distances between any pair of control images. The hollow circles represent the information distances between any pair of low control images, and the plus signs represent the information distances between any pair of high control images. The number of each subfigure index shows the ID number of the library plate, and the letter of that index represents one of the three different assay plates of a single library plate. 


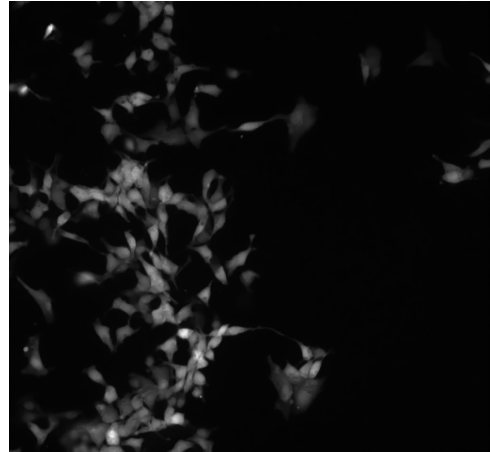

(a) Active Compound Sample

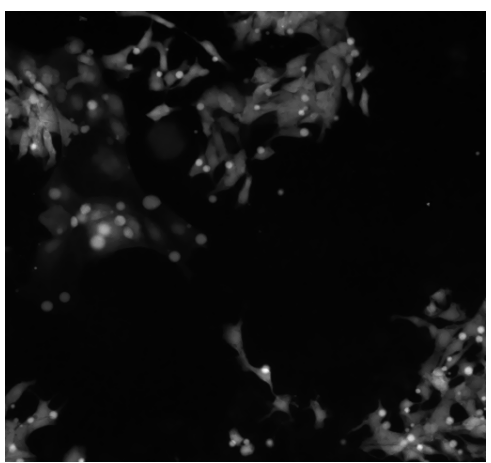

(b) Inactive Compound Sample

Fig. 5. Image samples taken randomly from active compounds (a) and inactive compounds (b). Fig. 5. a) is from well E11 of plate 1, which was identified as a "true" active compound; Fig.5 b) is from well E07 of plate 2, which was identified as a "true" inactive compound.

Table 2. Active compounds identification results of the biological verification experiment, the conventional analysis and the IDC method. This table shows the raw data of Table 1 in our paper. The checkmark indicates that the compound in the well is "active", while the backslash indicates that the compound in the well is "inactive".

\begin{tabular}{|c|c|c|c|c|c|}
\hline No. & Plate No. & Well No. & BIO & CON & IDC \\
\hline 1 & 1 & A06 & $\backslash$ & $\checkmark$ & $\checkmark$ \\
2 & 1 & E03 & $\backslash$ & $\backslash$ & $\backslash$ \\
3 & 1 & E07 & $\checkmark$ & $\backslash$ & $\backslash$ \\
4 & 1 & E08 & $\backslash$ & $\checkmark$ & $\checkmark$ \\
5 & 1 & E11 & $\checkmark$ & $\checkmark$ & $\checkmark$ \\
6 & 1 & F10 & $\checkmark$ & $\checkmark$ & $\checkmark$ \\
7 & 1 & G07 & $\checkmark$ & $\checkmark$ & $\checkmark$ \\
8 & 1 & G10 & $\checkmark$ & $\checkmark$ & $\checkmark$ \\
9 & 1 & H08 & $\checkmark$ & $\backslash$ & $\backslash$ \\
10 & 2 & A06 & $\checkmark$ & $\checkmark$ & $\checkmark$ \\
11 & 2 & A07 & $\checkmark$ & $\checkmark$ & $\checkmark$ \\
12 & 2 & A09 & $\checkmark$ & $\checkmark$ & $\checkmark$ \\
13 & 2 & B09 & $\backslash$ & $\backslash$ & $\backslash$ \\
14 & 2 & D06 & $\checkmark$ & $\checkmark$ & $\checkmark$ \\
15 & 2 & E07 & $\backslash$ & $\checkmark$ & $\backslash$ \\
16 & 2 & F07 & $\backslash$ & $\checkmark$ & $\backslash$ \\
17 & 2 & H08 & $\checkmark$ & $\checkmark$ & $\checkmark$ \\
18 & 3 & A04 & $\checkmark$ & $\checkmark$ & $\checkmark$ \\
19 & 3 & A10 & $\checkmark$ & $\checkmark$ & $\checkmark$ \\
20 & 3 & D02 & $\backslash$ & $\backslash$ & $\backslash$ \\
21 & 3 & D07 & $\checkmark$ & $\checkmark$ & $\checkmark$ \\
22 & 3 & G02 & $\checkmark$ & $\backslash$ & $\backslash$ \\
23 & 3 & G07 & $\checkmark$ & $\checkmark$ & $\checkmark$ \\
\hline
\end{tabular}

\begin{tabular}{|c|c|c|c|c|c|}
\hline No. & Plate No. & Well No. & BIO & CON & IDC \\
\hline 24 & 3 & H02 & $\backslash$ & $\backslash$ & $\backslash$ \\
25 & 3 & H06 & $\checkmark$ & $\checkmark$ & $\checkmark$ \\
26 & 3 & H09 & $\backslash$ & $\backslash$ & $\backslash$ \\
27 & 3 & F04 & $\backslash$ & $\backslash$ & $\backslash$ \\
28 & 4 & B04 & $\checkmark$ & $\checkmark$ & $\checkmark$ \\
29 & 4 & C04 & $\backslash$ & $\backslash$ & $\backslash$ \\
30 & 4 & D09 & $\backslash$ & $\backslash$ & $\backslash$ \\
31 & 4 & E05 & $\checkmark$ & $\checkmark$ & $\checkmark$ \\
32 & 4 & F04 & $\checkmark$ & $\checkmark$ & $\checkmark$ \\
33 & 5 & B03 & $\backslash$ & $\checkmark$ & $\checkmark$ \\
34 & 5 & B04 & $\checkmark$ & $\checkmark$ & $\checkmark$ \\
35 & 5 & C11 & $\checkmark$ & $\checkmark$ & $\checkmark$ \\
36 & 5 & D06 & $\backslash$ & $\backslash$ & $\backslash$ \\
37 & 5 & E02 & $\checkmark$ & $\checkmark$ & $\checkmark$ \\
38 & 5 & F07 & $\checkmark$ & $\checkmark$ & $\checkmark$ \\
39 & 5 & H09 & $\checkmark$ & $\checkmark$ & $\checkmark$ \\
40 & 5 & H10 & $\checkmark$ & $\checkmark$ & $\checkmark$ \\
41 & 6 & A02 & $\checkmark$ & $\checkmark$ & $\checkmark$ \\
42 & 6 & A03 & $\checkmark$ & $\checkmark$ & $\checkmark$ \\
43 & 6 & B02 & $\checkmark$ & $\checkmark$ & $\checkmark$ \\
44 & 6 & B03 & $\checkmark$ & $\checkmark$ & $\checkmark$ \\
45 & 6 & G03 & $\checkmark$ & $\checkmark$ & $\checkmark$ \\
46 & 6 & G04 & $\checkmark$ & $\checkmark$ & $\checkmark$ \\
\hline
\end{tabular}




\section{Conclusion}

Our methodology is based on information distance theory, and directly analyzes only cytoplasm images, with no need for DNA staining and image segmentation. Therefore, it avoids staining's cytotoxic/cytostatic problems and segmentation errors, and allows kinetic studies. Since it compresses images directly, there is no need to extract any features. In particular, it does not require a human intervention step, as is required in the conventional analysis, to obtain image information such as cell count and brightness.

Compared with the conventional analysis method, the IDC method achieves a higher F1 score. It is simpler to use, and acquires better results.

Acknowledgements. This work was funded by the Chinese Scholarship Council and was supported (in part) by an Ontario Ministry of Economic Development and Innovation (MEDI) grant.

\section{References}

1. Jaenisch, R., Young, R.: Stem Cells, the Molecular Circuitry of Pluripotency and Nuclear Reprogramming. Cell 132, 567-582 (2008)

2. Ding, S., Wu, T.Y.H., Brinker, A., Peters, E.C., Hur, W., Gray, N.S., Schultz, P.G.: Synthetic small molecules that control stem cell fate. PNAS 100, 7632-7637 (2003)

3. Ljosa, V., Carpenter, A.E.: Introduction to the Quantitative Analysis of Two-Dimensional Fluorescence Microscopy Images for Cell-Based Screening. Plos Computational Biology 5, 1-10 (2009)

4. Li, M., Vitanyi, P.: An introduction to Komogorov complexity and its applications. Springer, New York (1997)

5. Bennett, C.H., Gacs, P., Li, M., Vitanyi, P., Zurek, W.: Information Distance. IEEE Trans. Inform. Theory 44, 1407-1423 (1993)

6. Arbuchle, T., Balaban, A., Peters, D.K., Lawford, M.: Software documents: comparison and measurement. In: Proceeding 18th International Conference on Software Engineering \& Knowledge Engineering, Boston, USA, pp. 740-748 (2007)

7. Anë, C., Sanderson, M.J.: Missing the Forest for the Trees: Phylogenetic Compression and Its Implications for Inferring Complex Evolutionary Histories. J. Sys. Biol. 54, 146-157 (2005)

8. Campana, B.J.L., Keogh, E.J.: A Compression-Based Distance Measure for Texture. J. Statistical Analysis and Data Mining 3, 381-398 (2010)

9. Cerra, D., Mallet, A., Gueguen, L., Datcu, M.: Algorithmic Information Theory-Based Analysis of Earth Observation Images: An Assessment. J. IEEE Geoscience and Remote Sensing Letters 7, 8-12 (2010)

10. Chen, X., Francia, B., Li, M., Mckinnon, B., Seker, A.: Shared information and program plagiarism detection. IEEE Trans. Info. Theory 50, 1545-1550 (2004)

11. Cilibrasi, R., Vitänyi, P.M.B., de Wolf, R.: Algorithmic clustring of music based on string compression. J. Comput. Music 28, 49-67 (2004)

12. Cilibrasi, R., Vitänyi, P.M.B.: Clustering by compression. IEEE Trans. Knowledge \& Data Engineering 19, 370-383 (2007)

13. Cohen, A.R., Bjornsson, C.S., Temple, S., Banker, G., Roysam, B.: Automatic Summarization of Changes in Biological Image Sequences Using Algorithmic Information Theory. IEEE Trans. Pattern Analysis \& Machine Intelligence 31, 1386-1403 (2009) 
14. Cuturi, M., Vert, J.P.: The context-tree kernel for strings. Neural Networks 18, 1111-1123 (2005)

15. Benedetto, D., Caglioti, E., Loreto, V.: Language trees and zipping. Phys. Rev. Lett. 88, 48702 (2002)

16. Kocsor, A., Kertesz, F.A., Kajan, L., Pongor, S.: Application of compression-based distance measures to protein sequence classification: a methodology study. Bioinformatics 22, 407412 (2006)

17. Kirk, S.R., Jenkins, S.: Information theory-based software metrics and obfuscation. J. Systems and Software 72, 179-186 (2004)

18. Krasnogor, N., Pelta, D.A.: Measuring the similarity of protein structures by means of the universal similarity metric. Bioinformatics 20, 1015-1021 (2004)

19. Kraskov, A., Stögbauer, H., Andrzejak, R.G., Grassberger, P.: Hierarchical clustering using mutual information. Europhys. Lett. 70, 278-284 (2005)

20. Li, M., Badger, J.H., Chen, X., Kwong, S., Kearney, P., Zhang, H.Y.: An information-based sequence distance and its application to whole mitochondrial genome phylogeny. Bioinformatics 17, 149-154 (2001)

21. Nikvand, N., Wang, Z.: Generic Image Similarity Based on Kolmogorov Complexity. In: 17th IEEE International Conference on Image Processing, pp. 309-312. IEEE Press, Hong Kong (2010)

22. Otu, H.H., Sayood, K.: A new sequence distance measure for phy6logenetic tree construction. Bioinformatics 19, 2122-2130 (2003)

23. Pao, H.K., Case, J.: Computing entropy for ortholog detection. In: International Conference on Computational Intelligence, Istanbul, Turkey, pp. 89-92 (2004)

24. Parry, D.: Use of Kolmogorov distance identification of web page authorship, topic and domain. In: Workshop on Open Source Web Information Retrieval (2005)

25. Perkiö, J., Hyvärinen, A.: Modelling Image Complexity by Independent Component Analysis, with Application to Content-Based Image Retrieval. In: Alippi, C., Polycarpou, M., Panayiotou, C., Ellinas, G. (eds.) ICANN 2009, Part II. LNCS, vol. 5769, pp. 704-714. Springer, Heidelberg (2009)

26. Santos, C.C., Bernardes, J., Vitänyi, P.M.B., Antunes, L.: Clustering fetal heart rate tracings by compression. In: Proceeding 19th IEEE Internation Symposium Computer-Based Medical Systems, Salt Lake City, pp. 22-23 (2006)

27. Zhang, X., Hao, Y., Zhu, X.Y., Li, M.: Information Distance from a Question to an Answer. In: KDD, San Jose, pp. 12-15 (2007)

28. Rudin, L.I., Osher, S., Fatemi, E.: Nonlinear total variation based noise removel algorithms. Physica D 60, 259-268 (1992)

29. Panchal, R.G., Kota, K.P., Spurgers, K.B., Ruthel, G., Tran, J.P., Boltz, R.C., Bavari, S.: Development of High-Content Imaging Assays for Lethal Viral Pathogens. J. Biomol. Screen 15, 755-765 (2010)

30. Gall, D.L.: Mpeg: a video compression standard for multimedia application. Commun. ACM 34, 46-58 (1991)

31. http://www.perkinelmer.com/pages/020/cellularimaging/ products/acapella.xhtml 